\title{
Avaliação de biótipos de leiteiro com suspeita de resistência a herbicidas inibidores da ALS e Protox ${ }^{1}$
}

\author{
Evaluation of wild poinsettia biotypes with suspicion of resistant to ALS and Protox \\ inhibitors herbicides
}

\begin{abstract}
Alana Prigol' ${ }^{2}$; Leandro Galon ${ }^{3}$; César Tiago Forte ${ }^{4}$; Renato Kujawiski ${ }^{2}$ Germani Concenço ${ }^{5}$; Michelangelo Muzell Trezzi ${ }^{6}$; Ricardo Trevisol2 ${ }^{2}$; André Luiz Radünz ${ }^{7}$; Gismael Francisco Perin ${ }^{3}$
\end{abstract}

Resumo - Objetivou-se com o presente trabalho avaliar a confirmação da ocorrência de resistência de biótipos de leiteiro, oriundos de Rondônia e do Rio Grandes do Sul a herbicidas com diferentes mecanismos de ação, bem como determinar a dose dos herbicidas necessária para redução de $50 \%$ na massa seca das plantas $\left(\mathrm{GR}_{50}\right)$ e para o controle de $50 \%$ das plantas de cada unidade experimental $\left(\mathrm{C}_{50}\right)$. Para tanto foi instalado um experimento em casa de vegetação, em blocos casualizados, com quatro repetições. Os herbicidas utilizados foram: imazethapyr, chlorimuron-ethyl, cloransulam-methyl, saflufenacil, fomesafen e carfentrazone-ethyl. As doses utilizadas foram: $0 ; 0,5 ; 2 ; 8 ; 16 ; 32$ e 64 vezes a dose comercial. As variáveis avaliadas foram controle do leiteiro aos $07,14,21$ e 28 dias após a aplicação dos tratamentos (DAT) e também, aos 28 DAT determinou-se a massa seca das plantas. O biótipo do Rio Grande do Sul mostrou sensibilidade aos herbicidas testados, já o de Rondônia apresentou resistência cruzada aos inibidores de ALS (imazethapyr, chlorimuron e cloransulam) e suscetível aos inibidores de PROTOX (carfentrazone, fomesafen e saflufenacil).

Palavras-chaves: Euphorbia heterophylla L., Glycine max, plantas daninhas

\begin{abstract}
The objective of the present study was to evaluate the confirmation of occurrence of wild poinsettia resistance, coming from Rondônia and Rio Grande do Sul, to herbicides with different mechanisms of action, and to determine the dose of herbicides required for $50 \%$ reduction in the dry mass of plants $\left(\mathrm{GR}_{50}\right)$ and for the control of $50 \%$ of these plants in each experimental unit $\left(\mathrm{C}_{50}\right)$. Therefore, an experiment was conducted over greenhouse conditions, in randomized block design with four replications. The herbicides used were: imazethapyr, chlorimuron-ethyl, cloransulam-methyl, saflufenacil, fomesafen, and carfentrazone-ethyl. The doses used were: $0 ; 0.5$; $2 ; 8 ; 16 ; 32$ and 64 times the commercial dose. The variables evaluated were control of wild poinsettia at 7, 14, 21, and 28 days after treatments (DAT), and also at 28 DAT was determined the dry mass of plants. The Rio Grande do Sul biotype showed sensitivity to the herbicides tested,

\footnotetext{
${ }^{1}$ Recebido para publicação em 28/07/2014 e aceito em 28/06/2015.

${ }^{2}$ Acadêmico de Agronomia da Universidade Federal da Fronteira Sul (UFFS), Câmpus Erechim. Erechim, RS, BR.

${ }^{3}$ Professor da Universidade Federal da Fronteira Sul (UFFS), Câmpus Erechim. Erechim, RS, BR. Email: leandro.galon@uffs.edu.br. (*autor para correspondência).

${ }^{4}$ Mestrando do Programa de Pós-Graduação em Ciência e Tecnologia Ambiental da Universidade Federal da Fronteira Sul (UFFS), Câmpus Erechim. Erechim, RS, BR.

${ }^{5}$ Pesquisador da Embrapa Agropecuária Oeste (CPAO). Dourados, MS, BR.

${ }^{6}$ Professor da Universidade Tecnológica Federal do Paraná, Câmpus Pato Branco. Pato Branco, PR, BR.

${ }^{7}$ Bolsista DTI/FAPERGS/CAPES/UFFS, Câmpus Erechim/RS. Erechim, RS, BR.
} 
at meantime the Rondônia biotype presented cross-resistance to ALS-inhibitors (imazethapyr, chlorimuron, and cloransulam), and susceptibility to PROTOX-inhibitors (carfentrazone, fomesafen, and saflufenacil).

Keywords: Euphorbia heterophylla L., Glycine max, weeds

\section{Introdução}

As plantas daninhas interferem negativamente sobre a quantidade e a qualidade da produção agrícola (Paula et al., 2011). No Brasil, a competição da cultura da soja com o leiteiro (Euphorbia heterophylla L.) tem preocupado os agricultores, devido à dificuldade de manejo (Vargas et al., 2013) e os casos de resistência já registrados envolvendo esta espécie (Trezzi et al., 2005; Gelmini et al., 2001) de norte a sul do pais.

$\mathrm{O}$ aumento nos casos de resistência em plantas daninhas decorre, especialmente, da pressão de seleção devido à aplicação constante e abusiva de herbicidas com o mesmo mecanismo de ação (Trezzi et al., 2011; Costa e Rizzardi, 2014). Neste sentido, cabe destacar que a ampla utilização do método químico é consequência da estabilização do sistema de plantio direto, agregação de novas áreas produtivas, a crescente insuficiência de mão-deobra no campo, a elevada eficiência no controle e o alto rendimento das culturas (Christoffoleti et al., 2008; Oliveira Jr. et al., 2011).

Neste contexto, o leiteiro destaca-se como planta daninha preponderante, por ser de difícil controle, apresentar biótipos com resistência múltipla aos herbicidas inibidores da acetolactato sintase - ALS e protoporfirinogênio oxidase - PROTOX e ao mesmo tempo, essa planta daninha apresenta elevada habilidade competitiva ao conviver com as culturas (Kissman e Groth, 1999; Trezzi et al., 2005; Carvalho et al., 2010; Xavier et al., 2013). Para Meschede et al. (2002) a convivência de plantas de leiteiro com a soja, a partir do $17^{\circ}$ dia após sua emergência, resultou em perda diária de $5,15 \mathrm{~kg} \mathrm{ha}^{-1}$ na produtividade de grãos.

Considerando a importância atribuída ao controle químico de plantas daninhas na agricultura moderna e o impacto causado pela seleção de plantas daninhas resistentes a herbicidas, objetivou-se com o presente trabalho efetuar a confirmação da ocorrência de resistência de biótipos de leiteiro, oriundos dos estados de Rondônia (RO-2) e do Rio Grande do Sul (RS) a diferentes mecanismos de ação, bem como determinar a dose necessária para redução de $50 \%$ no acúmulo de massa seca das plantas $\left(\mathrm{GR}_{50}\right)$ e o controle de $50 \%$ das plantas de leiteiro $\left(\mathrm{C}_{50}\right)$.

\section{Material e Métodos}

O experimento foi instalado em casa da vegetação pertencente a Universidade Federal da Fronteira Sul (UFFS), Câmpus Erechim, Erechim/RS (27 $35^{\prime}$ S e $52^{\circ} 16^{\prime} \mathrm{W}$; altitude 762 m). Foram utilizadas sementes de Euphorbia heterophylla L. (leiteiro), coletadas em Erechim/RS, em local sem histórico de aplicação de herbicidas e em Cerejeiras/RO (13' $11^{\prime} \mathrm{S}$ de $60^{\circ} 51^{\prime} \mathrm{W}$; altitude de 257,8 m), em lavouras que se aplicava frequentemente herbicidas inibidores da ALS e da PROTOX, denominados, respectivamente, de RS (supostamente suscetível) e RO-2 (com suspeita de resistência).

Os biótipos foram semeados separadamente em 14/03/2014, em vasos plásticos perfurados, com capacidade para 3 $\mathrm{dm}^{3}$ preenchidos com substrato livre de qualquer contaminante e/ou propágulo de planta daninha, marca comercial Vivatto Pro 20. A irrigação foi realizada de forma regular durante toda a execução do experimento, a fim de garantir que a umidade do substrato se mantivesse próxima à capacidade de campo.

Após a emergência das plantas de leiteiro procedeu-se o desbaste, deixando-se duas plantas em cada unidade experimental. $\mathrm{O}$ delineamento experimental adotado foi o de 
blocos casualizados, com quatro repetições. Os herbicidas utilizados foram: imazethapyr, chlorimuron-ethyl, cloransulam-metlyl, saflufenacil, fomesafen e carfentrazone-ethyl. As doses utilizadas foram: $0 ; 0,5 ; 2 ; 8 ; 16 ; 32$ e 64 vezes a dose comercial. Para o controle de plantas daninhas infestantes da soja as doses registradas de imazethapyr, chlorimuron-ethyl, cloransulam-metlyl, saflufenacil, fomesafen e carfentrazone-ethyl são respectivamente de 100 , $20,40,35,250$ e $400 \mathrm{~g} \mathrm{ha}^{-1}$ de ingrediente ativo - i.a. (Rodrigues e Almeida, 2011). Desta forma, os tratamentos foram organizados em esquema fatorial $6 \times 7$.

A aplicação dos herbicidas foi efetuada aos 22 dias após a semeadura, quando as plantas de leiteiro se encontravam no estádio de quatro folhas. A aplicação foi realizada com pulverizador costal de precisão, pressurizado a $\mathrm{CO}_{2}$, equipado com duas pontas de pulverização tipo leque modelo 110.02 AD1, a qual resultou em uma taxa de aplicação de $200 \mathrm{~L} \mathrm{ha}^{-1}$.

As variáveis avaliadas foram: controle do leiteiro aos 07, 14, 21 e 28 dias após a aplicação dos tratamentos (DAT), atribuindo-se a nota de zero $(0 \%)$ aos tratamentos com ausência de controle e a nota cem (100\%) para controle total da planta daninha. Também, aos 28 DAT determinou-se a massa seca da parte aérea do leiteiro, sendo as plantas que não apresentaram $100 \%$ de controle, seccionadas rente ao solo e acondicionadas em sacos de papel e postas para secagem em estufa de circulação forçada de ar a temperatura de $65^{\circ} \mathrm{C}$ $\pm 5^{\circ} \mathrm{C}$ até atingirem massa constante. A $\mathrm{C}_{50}$ (dose necessária para controlar $50 \%$ das plantas do biótipo) foi calculada com os dados das avaliações de controle realizadas aos 28 DAT; a $\mathrm{GR}_{50}$ (dose necessária para inibição de $50 \%$ do acúmulo de massa das plantas) foi calculada com os dados das avaliações de massa seca coletada aos 28 DAT.

Os dados foram submetidos à análise de normalidade pelo teste de Shapiro-Wilk e de homogeneidade de variâncias pelo teste de Bartlett sendo quando necessários submetidos à transformação BoxCox (Conagin et al., 1993), até o alcance da homocedasticidade. Após ajustes os dados foram submetidos à análise de variância pelo teste $\mathrm{F}$ a $5 \%$ de probabilidade, sendo o efeito das doses estudadas por regressões lineares e não lineares. Para a escolha da regressão a ser empregada em cada caso, considerou-se o significado biológico, o maior ajuste do coeficiente de determinação $\left(\mathrm{R}^{2}\right)$ e o menor quadrado médio do resíduo, além da significância $(p \leq 0,05)$. As análises dos dados foram efetuadas no ambiente estatístico R ( R Development, 2012) e os gráficos elaborados no software SigmaPlot 12.3 (Systat, 2014). O eixo $\mathrm{X}$ dos gráficos foi apresentado em escala logarítmica; a dose " 0 " (zero) não é apresentada, sendo, porém, considerada na obtenção das curvas.

\section{Resultados e Discussão}

Houve diferenças entre os biótipos avaliados (RO-2 e RS) e os grupos químicos de herbicidas testados, inibidores de ALS e de PROTOX. De maneira geral, o biótipo RS foi sensível aos herbicidas quando comparado ao RO-2. Os herbicidas pertencentes ao grupo dos inibidores da ALS demonstraram menores efeitos sobre os biótipos, quando comparado aos inibidores de PROTOX. De acordo com Xavier et al. (2013) os herbicidas inibidores da ALS são responsáveis por cerca de $32 \%$ dos casos de resistência de plantas daninhas infestantes de culturas em todo o mundo. Desse modo, percebe-se que a ocorrência da resistência de plantas daninhas a estes herbicidas ocorre com maior frequência, quando comparado a outros mecanismos de ação. Esse fato deve-se ao uso repetitivo desses produtos na agricultura, a alta eficácia, a atividade residual no solo, alta frequência inicial de resistência da planta daninha, adaptabilidade ecológica do biótipo e as mutações pontuais na enzima ALS (Christoffoleti et al., 2008).

O biótipo RS não acumulou massa seca com a dose recomendada de saflufenacil, fomesafen e carfentrazone-ethyl e sob aplicação dos ALS no máximo com duas vezes a dose de 
imazethapyr, chlorimuron-ethyl e cloransulammethyl (Figura 1). Isto demonstra que este biótipo não é resistente aos herbicidas testados, o que é interessante do ponto de vista de manejo
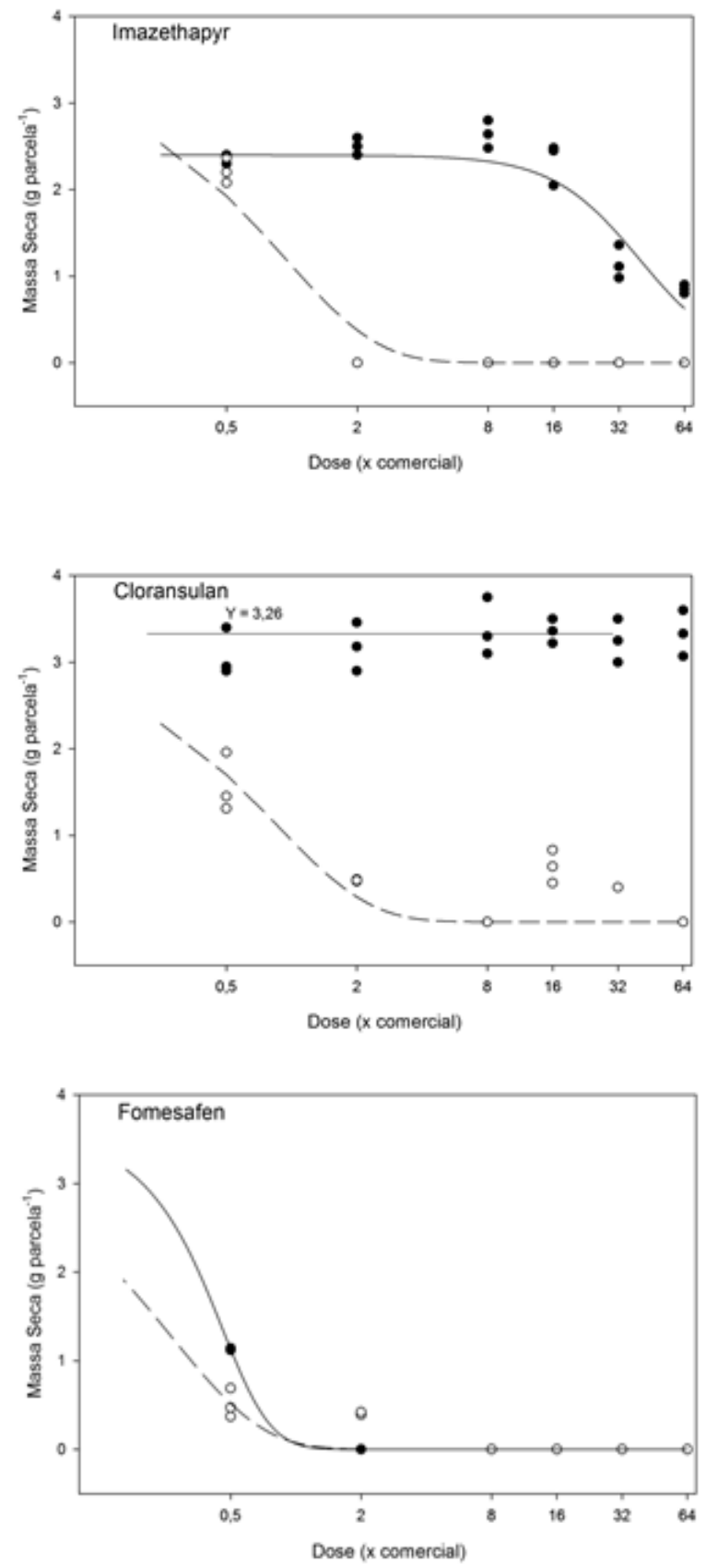

porque permite rotacionar grupos químicos, reduzindo a pressão de seleção sobre as plantas e consequentemente, o risco de resistência.
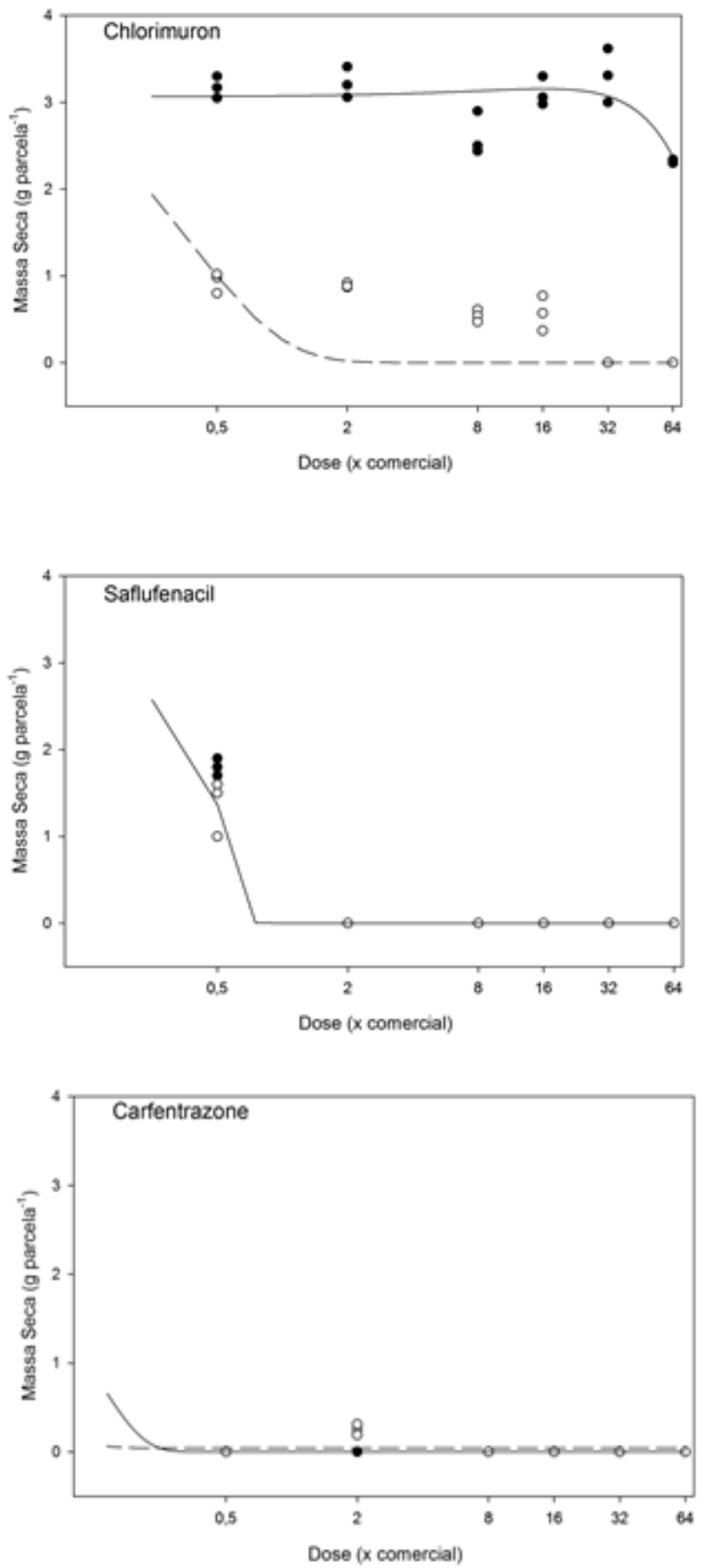

Eixo $\mathrm{X}$ apresentado em escala logarítmica

Figura 1. Acúmulo de massa seca nas plantas de leiteiro (Euphorbia heterophylla) dos biótipos RO-2 (suspeito de resistência •) e RS (suscetível ○), em função de herbicidas e doses, aos 28 dias após aplicação dos tratamentos (DAT). UFFS, Erechim/RS, 2014. 
Para Trezzi et al. (2005), o desenvolvimento de resistência múltipla de leiteiro a inibidores da ALS e PROTOX no Sudoeste do Paraná, está associado a alta pressão de seleção causada pelas aplicações de herbicidas inibidores da ALS, e, posteriormente, pelos inibidores de PROTOX, o que resultou na seleção de resistência a ambos os mecanismos de ação, em momentos distintos, mas na mesma população. Este fato também foi destacado por Xavier et al. (2013).

As avaliações realizadas sobre o biótipo RO-2 demonstram que este apresentou resistência aos herbicidas inibidores da enzima ALS (Figura 1), sendo necessário para imazethapyr e chlorimuron-ethyl, respectivamente a dose de 16 e 32 vezes a recomendada para provocar redução na massa seca acumulada. E para o cloransulam-methyl, mesmo com uma dose 64 vezes maior que a recomendada, não houve alteração na massa seca do leiteiro (Figura 1).

Cabe destacar que os dados se ajustam adequadamente aos modelos adotados, com exceção para o herbicida cloransulam-methyl onde não houve significância (Tabela 1).

Tabela 1. Equações, ajuste e significância das curvas de massa seca e controle de plantas de leiteiro (Euphorbia heterophylla), em função de herbicidas e doses, aos 28 dias após aplicação (DAA). UFFS, Erechim/RS, 2014.

\begin{tabular}{|c|c|c|c|c|c|c|c|}
\hline \multirow{2}{*}{ Herbicida } & \multicolumn{4}{|c|}{ Massa Seca } & \multicolumn{3}{|l|}{ Controle } \\
\hline & Biótipo & Equação & $\mathrm{R}^{2}$ & Sig. & Equação & $\mathrm{R}^{2}$ & Sig. \\
\hline \multirow{2}{*}{ Imazethapyr } & $\mathrm{RO}-2$ & $Y=2,4 /\left(1+(x / 39,6)^{2,2}\right)$ & 0,82 & $* *$ & $Y=56,6 /\left(1+(x / 32)^{-32}\right)$ & 0,98 & $* *$ \\
\hline & $\mathrm{RS}$ & $Y=3,33 e^{-1,1 x}$ & 0,97 & $* *$ & $Y=100 /\left(1+(x / 0,51)^{-15,4}\right)$ & 0,99 & $* *$ \\
\hline \multirow{2}{*}{ Chlorimuron } & $\mathrm{RO}-2$ & $Y=3,1-0,03 x^{2}$ & 0,47 & $*$ & $Y=11 /\left(1+(x / 57,8)^{-35}\right)$ & 0,96 & $* *$ \\
\hline & $\mathrm{RS}$ & $\mathrm{Y}=1687 /\left(1+\mathrm{e}^{(-(\mathrm{x}+2,29) /-0,37)}\right)$ & 0,86 & $* *$ & $Y=112 /\left(1+(x / 0,53)^{-0,44}\right)$ & 0,99 & $* *$ \\
\hline \multirow{2}{*}{ Cloransulam } & $\mathrm{RO}-2$ & $Y=3,26$ & --- & ns & $Y=11,6 /\left(1+(x / 58)^{-37}\right)$ & 0,99 & $* *$ \\
\hline & $\mathrm{RS}$ & $\mathrm{Y}=2962 /\left(1+\mathrm{e}^{(-(\mathrm{x}+5,79) /-0,84)}\right)$ & 0,89 & $* *$ & $Y=96 /\left(1+(x / 0,2)^{-1,1}\right)$ & 0,97 & $* *$ \\
\hline \multirow{2}{*}{ Saflufenacil } & $\mathrm{RO}-2$ & $\mathrm{Y}=2,97 /\left(1+(\mathrm{x} / 0,51)^{16,9}\right)$ & 0,99 & $* *$ & $Y=102 x /(0,2+x)$ & 0,99 & $* *$ \\
\hline & $\mathrm{RS}$ & $\mathrm{Y}=2,97 /\left(1+(\mathrm{x} / 0,51)^{16,9}\right)$ & 0,99 & $* *$ & $Y=102 x /(0,2+x)$ & 0,99 & $* *$ \\
\hline \multirow{2}{*}{ Fomesafen } & $\mathrm{RO}-2$ & $\mathrm{Y}=4,2 /\left(1+\mathrm{e}^{(-(\mathrm{x}-0,34) /-0,15)}\right)$ & 0,99 & $* *$ & $Y=100 /\left(1+(x / 0,28)^{-1,27}\right)$ & 0,99 & $* *$ \\
\hline & $\mathrm{RS}$ & $\mathrm{Y}=83 /\left(1+\mathrm{e}^{(-(\mathrm{x}+0,79) /-0,25)}\right)$ & 0,97 & $* *$ & $Y=100 /\left(1+(x / 0,28)^{-1,27}\right)$ & 0,99 & $* *$ \\
\hline \multirow{2}{*}{ Carfentrazone } & $\mathrm{RO}-2$ & $\mathrm{Y}=5,5 /\left(1+\mathrm{e}^{(-(\mathrm{x}-0,3) /-0,1)}\right)^{10}$ & 0,99 & $* *$ & $Y=102 x /(0,2+x)$ & 0,99 & $* *$ \\
\hline & $\mathrm{RS}$ & $\mathrm{Y}=0,04+3,8 /\left(1+\mathrm{e}^{(-(\mathrm{x}-0,05) /-0,02)}\right)$ & 0,98 & $* *$ & $Y=102 x /(0,2+x)$ & 0,99 & $* *$ \\
\hline
\end{tabular}

Avaliando o uso de herbicidas sobre biótipos de Euphorbia heterophylla, Gelmini et al. (2001) verificaram que mesmo aplicando-se dose oito vezes maior do que a recomendada de chlorimuron-ethyl e de imazethapyr, o biótipo resistente não foi controlado e não alterou a massa seca. Os mesmos autores relatam ainda que para o biótipo suscetível a dose recomendada mostrou-se eficaz.
As curvas de evolução (Figura 1) demonstram que o biótipo RS, quando submetido a herbicidas inibidores de ALS, apresentou redução no acúmulo de massa seca das plantas a partir de $0,5 \mathrm{x}$ a dose recomendada. Desta forma pode-se ainda constatar, a partir dos índices de resistência (Tabela 2), que o biótipo RO-2, em comparação com o RS, necessita doses de imazethapyr 43,6x e 61,9x 
maiores, respectivamente, para atingir a $\mathrm{GR}_{50} \mathrm{e}$ a $\mathrm{C}_{50}$. Para chlorimuron-ethyl e cloransulammethyl, as doses demandadas para alcançar a $\mathrm{GR}_{50}$ e a $\mathrm{C}_{50}$ do biótipo RO-2 não foram alcançadas com o intervalo testado, impossibilitando a obtenção dos valores reais do fator de resistência - RI (Tabela 2).

Assim, pode-se inferir sobre a ocorrência de resistência cruzada de leiteiro do biótipo RO-2 aos herbicidas inibidores da enzima ALS avaliados (Figura 1; Tabela 2). Embora seja verificado níveis distintos de resistência para cada princípio ativo, pode-se considerar que o biótipo RO-2 demonstrou alto nível de resistência, conforme preconizado também por Trezzi et al. (2005). A constatação da resistência no biótipo RO-2 e não no RS, possivelmente esteja associada as aplicações frequentes de herbicidas, as quais exerceram pressão de seleção sobre as plantas ao longo das gerações. O uso quase exclusivo de um mesmo herbicida tem aumentado os casos de resistência de plantas daninhas (Xavier et al., 2013). Convém destacar que o surgimento de novos casos de leiteiro resistente aos herbicidas inibidores de ALS e de PROTOX deve-se em grande parte por esses dois mecanismos de ação serem os mais usados no controle de plantas daninhas infestantes de culturas agrícolas. Também em Rondônia, apesar das áreas cultivadas serem relativamente recentes, se comparadas a outros estados brasileiros, tem apresentado casos de leiteiro resistente a ALS e de PROTOX (Xavier et al., 2013). Um dos motivos para que isso ocorra, é o fato de que, nesse estado, ainda os cultivos de culturas geneticamente modificadas, resistentes a herbicidas, não tenham participação efetiva nos cultivos. Desse modo, safra após safra os agricultores têm usado os herbicidas inibidores de ALS e PROTOX para o manejo de plantas daninhas o que favorece o surgimento de biótipos resistentes aos dois mecanismos de ação (Xavier et al., 2013).

Tabela 2. Dose do herbicida necessária para reduzir o crescimento das plantas de leiteiro (Euphorbia heterophylla) em $50 \%\left(\mathrm{GR}_{50}\right)$ ou para controlar $50 \%$ das plantas da parcela $\left(\mathrm{C}_{50}\right)$, aos 28 dias após aplicação dos tratamentos (DAT). UFFS, Erechim/RS, 2014.

\begin{tabular}{lcccccc}
\hline \multirow{2}{*}{ Herbicida } & \multicolumn{2}{c}{ Biótipo RO-2 } & \multicolumn{2}{c}{ Biótipo RS } & \multicolumn{2}{c}{$\mathrm{RI}_{\text {RO-2/RS }}$} \\
\cline { 2 - 7 } & $\mathrm{GR}_{50}$ & $\mathrm{C}_{50}$ & $\mathrm{GR}_{50}$ & $\mathrm{C}_{50}$ & $\mathrm{GR}_{50}$ & $\mathrm{C}_{50}$ \\
\hline Imazethapyr & $39,3 \mathrm{x}$ & $32,2 \mathrm{x}$ & $0,9 \mathrm{x}$ & $0,52 \mathrm{x}$ & 43,6 & 61,9 \\
\hline Chlorimuron & $>64 \mathrm{x}$ & $>64 \mathrm{x}$ & $0,35 \mathrm{x}$ & $0,33 \mathrm{x}$ & $>182$ & $>193$ \\
\hline Cloransulam & $>64 \mathrm{x}$ & $>64 \mathrm{x}$ & $0,8 \mathrm{x}$ & $0,22 \mathrm{x}$ & $>80$ & $>291$ \\
\hline Saflufenacil & $0,51 \mathrm{x}$ & $0,19 \mathrm{x}$ & $0,51 \mathrm{x}$ & $0,19 \mathrm{x}$ & 1 & 1 \\
\hline Fomesafen & $0,4 \mathrm{x}$ & $0,27 \mathrm{x}$ & $0,27 \mathrm{x}$ & $0,27 \mathrm{x}$ & 1,48 & 1 \\
\hline Carfentrazone & $0,14 \mathrm{x}$ & $0,19 \mathrm{x}$ & $0,12 \mathrm{x}$ & $0,19 \mathrm{x}$ & 1,16 & 1 \\
\hline $\mathrm{X}=$ vezes a dose comercial recomendada. & & & & &
\end{tabular}

Os resultados para controle do leiteiro do biótipo RS demonstram que aplicações de doses iguais ou superiores a $0,7 x, 32 x$ e $8 x$, respectivamente para imazethapyr, chlorimuron-ethyl e cloransulam-methyl (Figura 2) controlaram 100\% das plantas, sendo verificados bons ajustes dos dados (Tabela 2). Entretanto cabe considerar que o uso da metade da dose recomendada provocou controle de 40, 52 e $66 \%$ das plantas de leiteiro (RS), respectivamente para o imazethapyr, chlorimuron-ethyl e cloransulam-methyl (Figura 2).

O biótipo RO-2 sob aplicação dos inibidores de ALS, apresentou baixo controle, sendo a $\mathrm{C}_{50}$ de $32,2 x$ para imazethapyr, e não 
tendo sido alcançada, mesmo com a maior dose testada, 64x, para chlorimuron-ethyl $\mathrm{e}$ cloransulam-methyl (Tabela 2), indicando alto nível de resistência do biótipo. Assim, verificou-se para o biótipo RO-2 que a dose
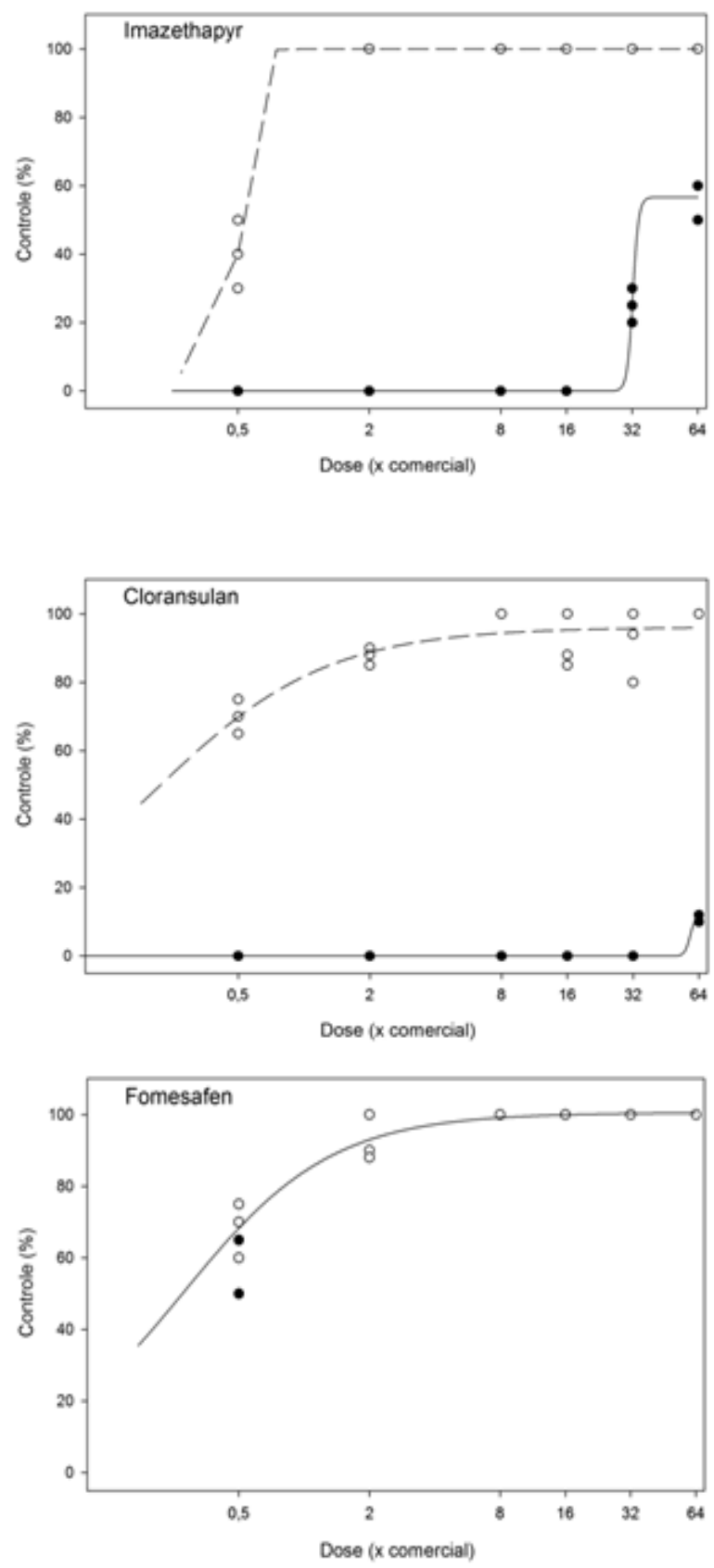

máxima testada $(64 \mathrm{x})$ resultou em controle de 53,12 e $10 \%$ das plantas, respectivamente para imazethapyr, chlorimuron-ethyl e cloransulammethyl (Figura 2).
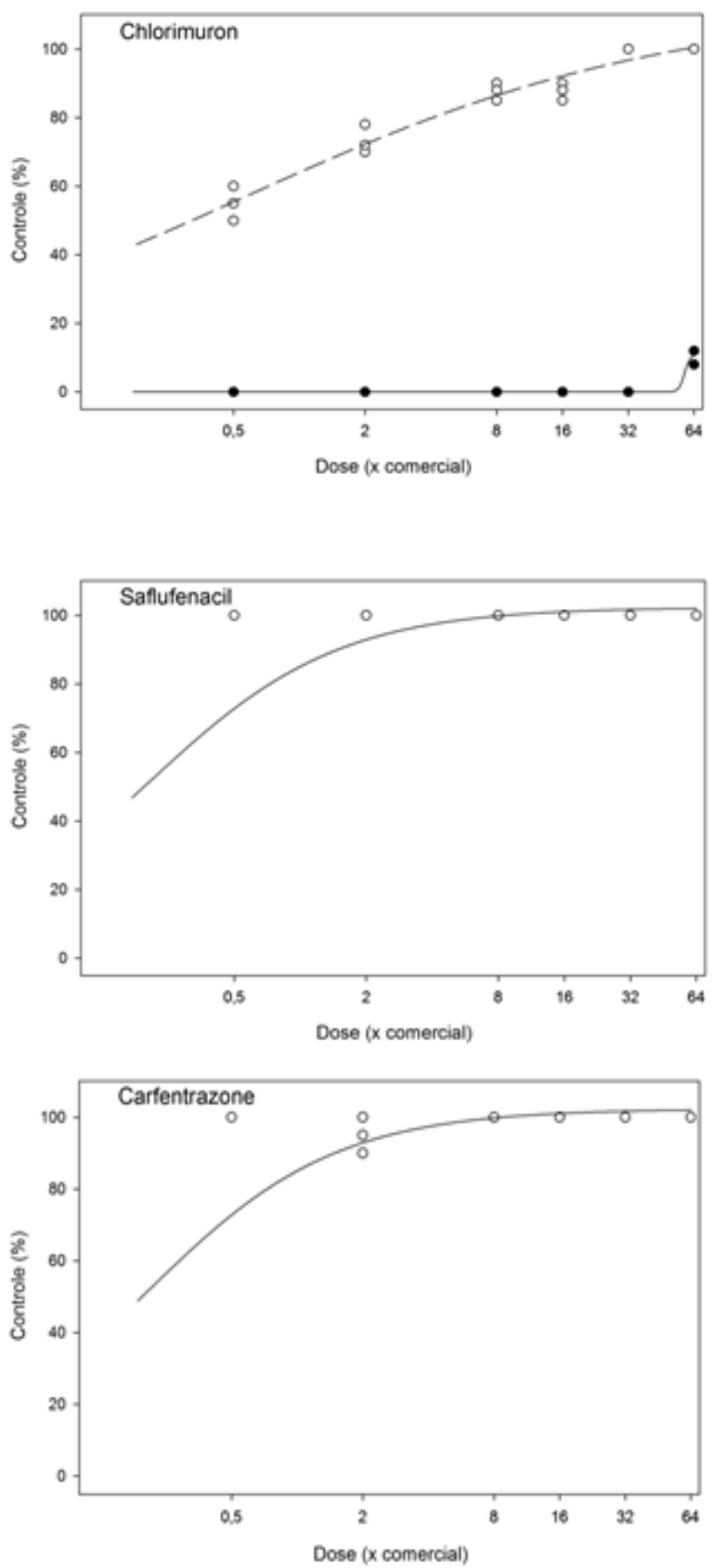

Eixo $\mathrm{X}$ apresentado em escala logarítmica

Figura 2. Controle (\%) de leiteiro (Euphorbia heterophylla) em biótipos RO-2 (suspeito de resistência •) e RS (suscetível ○), em função de herbicidas e doses, aos 28 dias após aplicação dos tratamentos (DAT). UFFS, Erechim/RS, 2014. 
Trezzi et al. (2011) observaram aumento no controle de biótipos de leiteiro com a elevação da dose de herbicidas inibidores de ALS e PROTOX, sendo o controle do leiteiro mais acentuado para biótipos suscetíveis que os resistentes, em uma mesma dose avaliada. A resistência dos biótipos a ALS, segundo Christoffoleti et al. (2008) deve-se a uma modificação do gene responsável pela codificação da enzima, tornando a planta resistente aos herbicidas inibidores dessa enzima, que, por conseguinte altera a sequência de aminoácidos, não permitindo que herbicidas como o imazethapyr, chlorimuron-ethyl e cloransulam-methyl consigam provocar a inibição da enzima ALS.

Comparando-se os fatores de resistência (RI) do biótipo RO-2 em relação ao RS, para $\mathrm{GR}_{50}$ e $\mathrm{C}_{50}$, observou-se que o grupo das imidazolinonas foi mais eficiente no controle do leiteiro que o das sulfoniluréias (Tabela 2). Destaca-se que o cloransulam-methyl onde o RI obtido para o biótipo RO-2 foi 291x superior ao biótipo RS (Tabelas 1 e 2).

A utilização dos herbicidas pertencentes ao grupo dos inibidores da enzima PROTOX, foram eficientes tanto na redução da massa seca das plantas (Figura 1) como no seu controle (Figura 2) de leiteiro do biótipo RO-2. Os mesmos resultados foram identificados por Gelmini et al. (2001), os quais avaliaram herbicidas pertencentes aos inibidores de PROTOX, tradicionalmente utilizados na cultura da soja, e constataram que estes exerceram controle eficaz em biótipos de Euphorbia heterophylla.

Para os herbicidas inibidores de PROTOX (saflufenacil, fomesafen e carfentrazone-ethyl), foi constatado similaridade de controle entre biótipos, sendo que houve aumento do percentual em função do incremento na dose (Figura 2). Observou-se para os herbicidas saflufenacil, fomesafen e carfentrazone-ethyl, respectivamente redução de $50 \%$ na massa seca das plantas $\left(\mathrm{GR}_{50}\right)$, em média, com o uso de 0,$51 ; 0,34$ e 0,13 vezes a dose recomendada e controle total de $50 \%$ das plantas de leiteiro $\left(\mathrm{C}_{50}\right)$, em média, com o uso de 0,$19 ; 0,27$ e 0,19 vezes a dose recomendada (Tabela 2).

No que tange a diferença de eficiência sobre os biótipos RO-2 e RS foi observada a necessidade de doses 48 e $16 \%$ maiores de fomesafen e carfentrazone-ethyl para supressão de $50 \%$ da massa seca das plantas de leiteiro do biótipo RO-2 comparado ao RS. Entretanto, considerando que a supressão de $50 \%$ no acúmulo de massa seca do biótipo RO-2 foi alcançada com metade da dose recomendada (Tabela 2), destaca-se a eficiência no uso destes herbicidas.

\section{Conclusões}

O biótipo RS mostrou sensibilidade aos herbicidas testados, já o RO-2 apresentou resistência cruzada aos inibidores de ALS (imazethapyr, chlorimuron e cloransulam) e suscetível aos inibidores de PROTOX (carfentrazone, fomesafen e saflufenacil).

\section{Agradecimentos}

Ao Conselho Nacional de Desenvolvimento Científico e Tecnológico $(\mathrm{CNPq})$ e à Fundação de Amparo à Pesquisa do Estado do Rio Grande do Sul (FAPERGS), pelo auxílio financeiro à pesquisa (Processos n. 482144/2012-2/CNPq e 12/2265-3/FAPERGS) e pelas concessões de bolsas.

\section{Referências}

CARVALHO, L.B. et al. Interferência de Euphorbia heterophylla no crescimento e acúmulo de macronutrientes da soja. Planta Daninha, v.28, n.1, p.33-39, 2010.

CHRISTOFFOLETI, P.J. et al. Aspectos de resistência de plantas daninhas a herbicidas. 3 ed. Piracicaba: Associação Brasileira de Ação à Resistência de Plantas Daninhas HRAC-BR, 2008, 120p.

CONAGIN, A. et al. Efeito da falta de normalidade em testes de homogeneidade das 
variâncias. Bragantia, v.52, n.2, p.173-180, 1993.

COSTA, L.O.; RIZZARDI, M.A. Resistance of Raphanus raphanistrum to the herbicide metsulfuron-methyl. Planta Daninha, v.32, n.1, p.181-187, 2014.

GELMINI, G.A. et al. Resistência de biótipos de Euphorbia heterophylla L. aos herbicidas inibidores da enzima ALS utilizados na cultura de soja. Bragantia, v.60, n.2, p.93-99, 2001.

KISSMANN, K.G.; GROTH, D. Plantas infestantes e nocivas. 2.ed. São Paulo: BASF, 1999. Tomo II. 978 p.

MESCHEDE, D.K. et al. Período crítico de interferência de E. heterophylla na cultura da soja sob baixa densidade de semeadura. Planta Daninha, v.20, n.3, p.381-387, 2002.

OLIVEIRA JR., R.S.; CONSTANTIN J.; INOUE, M.H. Biologia e Manejo de Plantas Daninhas, Editora OMNIPAX, 348p, 2011.

PAULA, J.M. et al. Manejo de Conyza bonariensis resistente ao herbicida glyphosate. Planta Daninha, v.29, n.1, p.217-227, 2011.

R DEVELOPMENT CORE TEAM. R: A language and environment for statistical computing. Vienna, Austria: R Foundation for Statistical Computing, 2012.

RODRIGUES, B.N.; ALMEIDA, F.R. Guia de herbicidas. $5^{a}$ Edição. Londrina: Edição dos Autores, 2011. 697p.

SYSTAT. SigmaPlot 12.3. Disponível em: http://sigmaplot.com. Acesso em 10/01/2013.

TREZZI, M.M. et al. Multiple resistance of acetolactate synthase and protoporphyrinogen oxidase inhibitors in Euphorbia heterophylla biotypes. Journal of Environmental Science and Health, Part B, v.40, n. 1, p.101-109, 2005.

TREZZI, M.M. et al. Teste rápido de imersão foliar de Euphorbia heterophylla para confirmação de resistência a herbicidas inibidores da Protox e da ALS. Planta Daninha, v.29, n.4, p.901-912, 2011.
VARGAS, L. et al. Práticas de manejo e a resistência de Euphorbia heterophylla aos inibidores da ALS e tolerância ao glyphosate no Rio Grande do Sul. Planta Daninha, v.31, n.2, p.427-432, 2013.

XAVIER, E. et al. Acetolactate synthase activity in Euphorbia heterophylla resistant to ALS- and protox- inhibiting herbicides. Planta Daninha, v.31, n.4, p.867-874, 2013. 